\title{
ECG-Based Heartbeat Classification using Machine Learning: Survey
}

\author{
Komal S. Jaisinghani ${ }^{1}$ and Sandeep Malik ${ }^{2}$ \\ ${ }^{1}$ Ph. D. Scholar at Oriental University, Indore \& Assist. Professor at SVPCET, Nagpur, India \\ ${ }^{2}$ Associate Professor, Oriental University, Indore, India
}

\section{ABSTRACT}

Cardiovascular diseases (CVDs) affecting millions of people around the world. Classification of heartbeat is very important step to determine cardiac functionality. An electrocardiogram (ECG), (a graphical representation of heart signals) is used to measure the electric signals of the heart and is widely used for detecting any abnormality lies within. By analyzing and studying the electrical signals generated from ECG with the help of electrodes, it is possible to detect some of the problems in heart. There are many types of classifiers available for Heartbeat classification. However in this paper we survey the methods used for automatic ECG-based heartbeat classification by discussing pre processing, Electrocardiogram dataset, feature extraction and types of classifiers available for automatic heartbeat classification.

KEY WORDS: ECG, HEARTBEAT CLASSIFICATION, CLASSIFIER, ECG DATASET.

\section{INTRODUCTION}

Cardio vascular diseases affect millions of people throughout the world every year. If your Heart is not working according to Normal beats then it indicates that there is a cardiovascular disease. Heartbeats not properly working means there is a common heart related issue called as arrhythmia. There are many reasons for arrhythmia, if there is an irregular conduction and formation of electrical signal, which may results in changing the heartbeat impulse shapes and it may create problem in normal functioning of heartbeat. The process or method of finding and differentiating arrhythmia can be very complex for a human being because sometimes it is necessary and important to analyze each heartbeat impulse of the records generated by ECG machine,

\section{ARTICLE INFORMATION}

*Corresponding Author: 10584.komal@gmail.com

Received 14th Oct 2020 Accepted after revision 23rd Dec 2020

Print ISSN: 0974-6455 Online ISSN: 2321-4007 CODEN: BBRCBA

Thomson Reuters ISI Web of Science Clarivate Analytics USA and Crossref Indexed Journal

\section{Clarivate
Analytics}

NAAS Journal Score 2020 (4.31)

A Society of Science and Nature Publication,

Bhopal India 2020. All rights reserved.

Online Contents Available at: http//www.bbrc.in/

Doi: http://dx.doi.org/10.21786/bbrc/13.14/94 acquired by a holter monitor for example, during hours, or even for days.

Apart from this, there are the chances of human mistakes during the analysis of ECG records, due to health conditions of patient. A substitute is to use techniques based on computation for human less and machine oriented classification. A fully automatic and mechanized system for heartbeat classification from impulses acquired by an ECG machine can be bifurcated in 5 steps: - (1) Pre-processing of ECG impulses (2) heart beat segmentation (3) Feature finding (4) Feature selection and (5) Classification of arrhythmia. In each of these 5 steps, an action is performed and the final target is to discriminate/identify the type of heartbeat.

Related Work: The function of electrocardiogram (ECG) is to measure the electrical activity of the heart and to detect heart diseases. It is possible to diagnose whether there are any anomalies in the heart by examining the electrical signal of each heartbeat produced by the ECG study. Till now many researchers developed different classifiers which are used for automatic ECG-based heartbeat classification. In survey of ECG, author gives overview of different methods used for ECG-based automatic heartbeat classification. [Eduardo José da S. Luza, 2016].

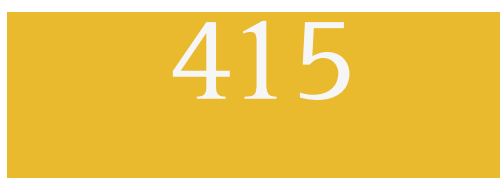


A hybrid model was proposed which is used for differentiation of cardiac arrhythmias and also used to select their features in optimum way. Genetic Algorithm (GA) was utilized for determination of the element ideally and the Decision Tree with the C4.5 calculation was applied for highlight finding and to separate and prepare the model. In this research author has utilized the GA and DT strategies as another blend (Hybrid) model to take care of the arrhythmia characterization issue and to evaluate the presentation of the proposed model, UCI arrhythmia dataset was utilized to figure exact outcomes, affectability, particularity, and normal Sen-Spec measurements. [Mehdi Ayar, 2018].

Figure 1: A diagram of the arrhythmia classification system

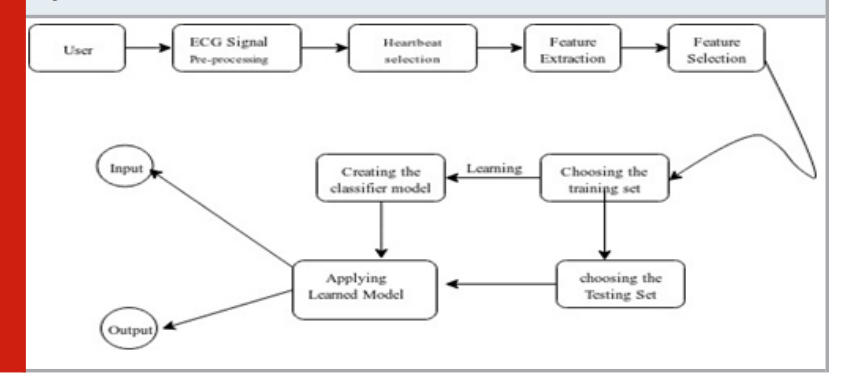

Ensemble method for ECG based heartbeat classification was proposed for this. Ensemble method is a metaalgorithm which is used to make strong classifiers that were based on a consortium of weak classifiers. In this work, author has explored some ensemble classifiers on UCI Arrhythmia Dataset to arrange the heartbeat data. In this work, XGBoost, Random Forest ensemble classifiers along with classic Logistic Regression was used for comparing results. The author has also designed Voting Classifier which are based on ensemble voting meta-algorithm and the above 3 built-in classifiers. The said design outperforms even well tuned XGBoost and Random Forest classifiers. The best prediction accuracy achieved by the Voting Classifier was 76\% in multiclass classification problem [Rui Duan, 2019].

Conventional Neural network method was proposed for heart rate deviation detection because of the automated feature processing layers, CNN does not require extra work relative to conventional machine learning approaches. In the classification of heartbeat problems, CNN proved extremely competitive. [Dokur ,Wang 2020]. Time-domain aspect produced from the 1-lead Electrographic machine were intentionally opted to make them satisfied, and the classification process of the heartbeat by utilizing RF was reasonably assessed by succeeding; the AAMI guidelines and the paradigm of the person who is suffering. The results obtained were best with the help of best 6 most enlightened attitudes and a 40-trees RF classifier. The assessment results based on the arrhythmia database of MIT-BIH resulted in an overall precision of $96.14 \%$ with definite F1-scores of 73.06\%, 97.97\% and $90.85 \%$ for the SVEB, VEB and NB, classes, appropriately. [Jose Francisco Saenz-Cogollo , 2020]

A comparison based on a Deep Neural Network (DNN) for the mechanized division of irregular ECG beats, separated from regular ones. It has been established by utilizing the free and open source software library, the pre-written professional quality codes from Google, which comprises of 7 concealed layers, with Five, Ten, Thirty, Fifty, Thirty, Ten and Five neurons, appropriately. The free and open source software library is to be utilized in the training or examining mode of the DNN [G. Sannino, 2018]. A fast, efficient ECG arrhythmia differentiator build on an easy intellectual capacity motivated by mechanized study method termed as Echo State Networks. The heart rate differentiator is analyzed over 2 ECG data contents, the AR, the AHA and Massachusetts Institute of TechnologyBeth Israel Hospital (MIT-BIH).

In the Massachusetts Institute of Technology-Beth Israel Hospital (MIT-BIH) data content, the classification/ differentiation method gives $92.70 \%$ perceptivity and $86.1 \%$ of practical expected figure for the ventricular ectopic heart beats, using the 1-lead II [Miguel C. Soriano, 2019]. Automatic Cardiac Arrhythmia detection using abnormal ECG was investigated. Data classifier by using deep learning model was used and applied on wearable ECG monitoring devices. Android terminal module was used. This study relates with artificial intelligence (AI), deep learning, cloud computing. Experimental results gives better reliability, less cost, good accuracy of deep learning models [Xin Gao, 2019 ].

\section{Proposed Model:}

A. ECG Raw Data: Generally for ECG classification, the dataset which is used is from MIT-BIH dataset. This database is generally used as standard dataset for detecting cardiac problem. The MIT-BIH database consisting of 48 ECG records which was obtained from 47 subjects which was sampled at frequency $360 \mathrm{~Hz}$.

B. Pre-processing of ECG Data: Preprocessing of ECG data is very important and challenging to remove noise from ECG data and produce a clear ECG data. There are different noise available with ECG data like muscle noise, impulsive noise, frequency noise. Many different methods are available to remove these noises such as wavelet coefficient threshold, FIR filter, median filter and Gaussian approach. It is necessary to select proper de-noising algorithm in preprocessing of ECG data.

C. Feature Extraction \& Feature Selection: Feature extraction contains most of the important data from original data. Wavelet Transform method is generally used for feature extraction of ECG Data. Morphological and temporal features are extracted from ECG data. For selection of various features, the techniques which are used are as follows; Grey Wolf Optimizer (GWO), Genetic Algorithm (GA), and Particle Swarm Optimization (PSO). 
D. Classifier used for classification: Different classifiers are used for heartbeat classification such as support vector machine (SVM), Convolution Neural Network
(CNN), Artificial Neural Network (ANN), Random Forest Classifier, Hierarchical Classification, Echo sate network classifier, Deep Neural Network.

\begin{tabular}{|c|c|c|c|c|}
\hline Sr. No. & Model of Classifier & Characteristics & Dataset & $\begin{array}{c}\text { Performance } \\
\text { Metrics }\end{array}$ \\
\hline 1 & $\begin{array}{c}\text { Hybrid Model with Genetic } \\
\text { Algorithm and } \\
\text { Decision Trees Used. }\end{array}$ & $\begin{array}{c}\text { Genetic Algorithm (GA) was used } \\
\text { for feature selection. For classification } \\
\text { and training features Decision } \\
\text { Tree with the C4.5 algorithm } \\
\text { was applied }\end{array}$ & $\begin{array}{l}\text { UCI arrhythmia } \\
\text { dataset }\end{array}$ & $\begin{array}{ll}\text { Accuracy:- } & 86.96 \% \\
\text { Sensitivity:- } & 88.88 \%\end{array}$ \\
\hline 2 & $\begin{array}{l}\text { Ensemble Classifier } \\
\text { XGBoost and } \\
\text { Random Forest used }\end{array}$ & $\begin{array}{l}\text { XGBoost and Random } \\
\text { Forest Ensemble classifiers were } \\
\text { used, and the classic Logistic } \\
\text { Regression was used for } \\
\text { comparison. Voting Classifier } \\
\text { was built based on the } \\
\text { ensemble voting meta algorithm }\end{array}$ & $\begin{array}{l}\text { UCI } \\
\text { arrhythmia } \\
\text { dataset }\end{array}$ & $\begin{array}{l}\text { Accuracy :- 76\% } \\
\text { by the Voting } \\
\text { Classifier }\end{array}$ \\
\hline 3 & $\begin{array}{l}\text { Convolution neural } \\
\text { network with Walsh } \\
\text { functions used [4] }\end{array}$ & $\begin{array}{l}\text { In this research, Walsh function } \\
\text { (WF) was used and discrepancies } \\
\text { of converting } 1 \mathrm{D} \text { signals to } 2 \mathrm{D} \\
\text { images have been investigated. }\end{array}$ & $\begin{array}{l}\text { MIT-BIH } \\
\text { arrhythmia } \\
\text { dataset }\end{array}$ & Accuracy :- 99\% \\
\hline 4 & $\begin{array}{c}\text { An Improved } \\
\text { Convolutional } \\
\text { Neural Network }\end{array}$ & $\begin{array}{l}\text { For ECG classification Advances } \\
\text { Convolutional neural network } \\
\text { (CNN) model was used. If the proposed } \\
\text { improved CNN model can be trained } \\
\text { properly then it works as a tool to } \\
\text { automatically identify different kinds } \\
\text { of arrhythmia from ECG. }\end{array}$ & $\begin{array}{l}\text { MIT-BIH } \\
\text { arrhythmia } \\
\text { dataset }\end{array}$ & $\begin{array}{l}\text { Accuracy 99.06\% [5]. } \\
\text { Accuracy 99.43\% [6]. }\end{array}$ \\
\hline 5 & $\begin{array}{l}\text { Random Forests } \\
\text { Classifier used }\end{array}$ & $\begin{array}{l}\text { In this research Random } \\
\text { forest classifier was used for } \\
\text { selection of informative features. }\end{array}$ & $\begin{array}{l}\text { AAMI guidelines } \\
\text { MIT-BIH dataset }\end{array}$ & Accuracy of $96.14 \%$ \\
\hline 6 & $\begin{array}{l}\text { Hierarchical } \\
\text { Classification } \\
\text { approach was } \\
\text { proposed. }\end{array}$ & $\begin{array}{l}\text { Hierarchical approach provided an } \\
\text { improvement in classification } \\
\text { performance of } \mathrm{N} \text { and } \\
\text { SVEB classes. }\end{array}$ & MIT-BH dataset & \\
\hline 7 & $\begin{array}{l}\text { Echo State Network } \\
\text { Classifier was used. }\end{array}$ & $\begin{array}{l}\text { Combination of ensemble } \\
\text { helps to train classifier } \\
\text { with high speed. }\end{array}$ & MIT-BH dataset & Sensitivity is $92.7 \%$ \\
\hline
\end{tabular}

\section{E. Performance Parameters:}

Performance parameters are generally Sensitivity, Specificity and accuracy. These parameters are achieved from the classification result.

- SEV (Sensitivity): The sensitivity test is to calculate number of patients with disease.

- SPV (Specificity): The specificity test is to find number of patients without the disease.

- TPV True positive values : Positive test means patient is suffering from disease

- FPV False positive : Positive test means the patient is not suffering from disease

- TNV True negative: Negative test means the patient is not suffering from disease
- FNV False negative: Negative test but the patient has the disease.

Equations of SE and SP:

$$
\begin{aligned}
& \mathrm{SEV}=\frac{\mathrm{TPV}}{\mathrm{TPV}}+\mathrm{FNV} * 100 \\
& \mathrm{SPV}=\frac{\mathrm{TNV}}{\mathrm{TNV}}+\mathrm{FPV} * 100
\end{aligned}
$$

- CC: Correct Classification (Accuracy) is computed as below: 
Figure 2: Proposed Model

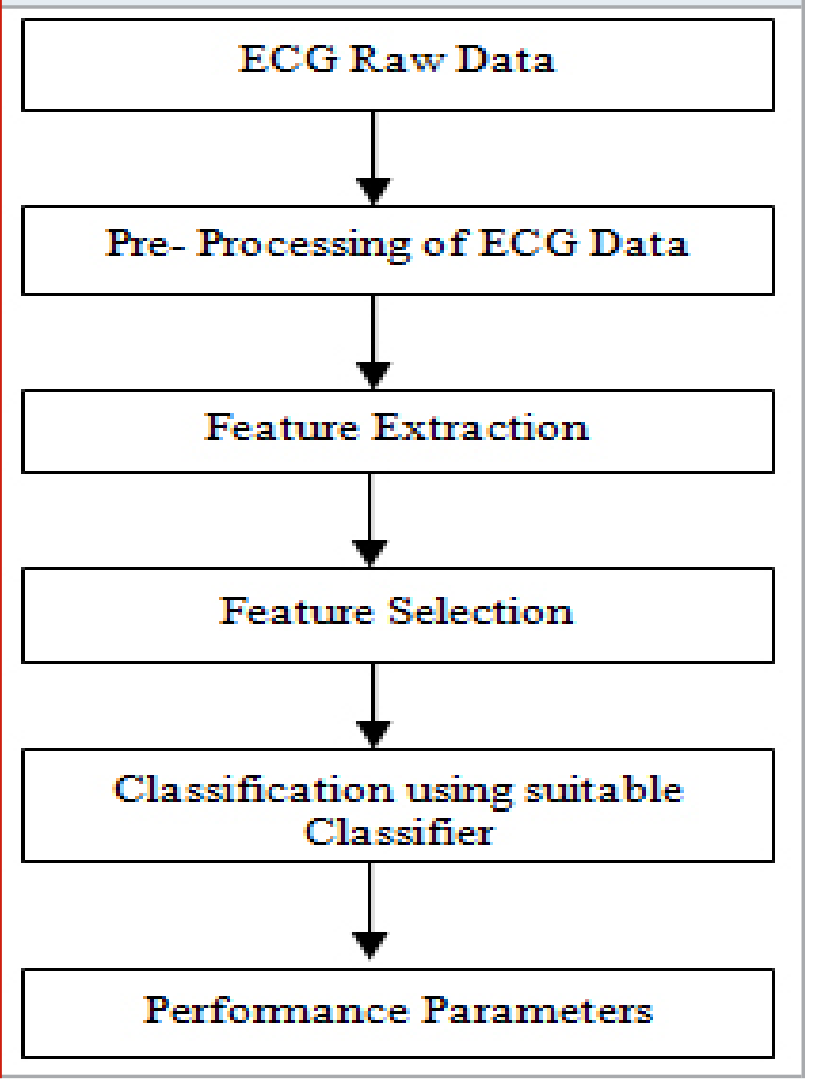

CONCLUSION

We have reviewed various methods used for automatic ECG based heart rate classification in this review article. For arrhythmia detection, the standard defined classifiers such as neural network and SVM are found to be accurate. Using morphological and time frequency based aspects; the precision rate of more than 99 percent is achieved. Recent research shows that deep learning methods are successful in terms of computational complexity and accuracy compatibility compared to the regular classifiers.

\section{REFERENCES}

A.H., Ribeiro, M.H., Paixão, G.M.M. et al., "Automatic diagnosis of the 12-lead ECG using a deep neural network", Nat Commun 11, 1760 (2020). https://doi. org/10.1038/s41467-020-15432-4

C. Ganesh Babu M. Gowri Shankar, "An Exploration of ECG Signal Feature Selection and Classification using Machine Learning Techniques,"International Journal of Innovative Technology and Exploring Engineering (IJITEE), vol. 9, no. 3, January 2020. https://www. researchgate.net/deref/http $\% 3 \mathrm{~A} \% 2 \mathrm{~F} \% 2 \mathrm{Fdx}$.doi. org\%2F10.35940\%2Fijitee.C8728.019320

Dokur, Z., Ölmez, T., "Heartbeat classification by using a convolutional neural network trained with Walsh functions", Neural Comput \& Applic 32, 12515-12534
(2020). https://doi.org/10.1007/s00521-020-04709-w Eduardo J. da S. Luz and, "Evaluating a hierarchical approach for heartbeat classification from ECG,"Int. J. Bioinformatics Research and Applications, vol. 13, no. 2, pp. 146-160, 2017.

Eduardo José da S.Luza,William Robson Schwartzb, Guillermo Cámara-Cháveza, David Menottiac, "ECGbased heartbeat classification for arrhythmia detection: A survey", journal of Computer Methods and Programs in BiomedicineVolume 127, ELSEVIER,April 2016, pp. 144-164. https://doi.org/10.1016/j.cmpb.2015.12.008 Fajr Ibrahem Alarsan 1 and Mamoon Younes2, "Analysis and classification of heart diseases using heartbeat features and machine learning algorithms,"Journal of Big Data, Springer Open, 2019. https://doi.org/10.1186/ s40537-019-0244-X

G. Sannino, G. De Pietro "A deep learning approach for ECG-based heartbeat classification for arrhythmia detection,” journal of Future Generation Computer Systems ELSEVIER, pp. 446-455, 2018. https://doi. org/10.1016/j.future.2018.03.057

HONGXING LIU2 XUEXIANG XU1, "ECG Heartbeat Classification Using Convolutional Neural Networks," IEEE Access, 2017, Volume: 8 pp. 8614 - 8619. https:// doi.org/10.1109/ACCESS.2020.2964749

Jose Francisco Saenz-Cogollo and Maurizio Agelli, ” Investigating Feature Selection and Random Forests for Inter-Patient Heartbeat Classification,"MPDI Algorithms 2020, March 2020.

Mehdi Ayar, Saeed Sabamoniri, "An ECG-ased feature selection and heartbeat classification model using a hybrid heuristic algorithm”, journal of Informatics in Medicine UnlockedVolume 13, ELSEVIER, 2018, pp.167175 https://doi.org/10.1016/j.imu.2018.06.002

Miguel C. Soriano and Silvia Ortín Miquel Alfaras , "A Fast Machine Learning Model for ECG-Based Heartbeat Classification and Arrhythmia Detection,"Frontiers in Physics, vol. 7, july 2019.

Rui Duan, Sabah Mohammed and Jinan Fiaidhi, "ENSEMBLE METHODS FOR ECG-BASED HEARTBEAT CLASSIFICATION," International Journal of Control and Automation, vol. 12, no. 4, pp. 29-46, 2019. http/dx.doi. org/10.33832/ijca.2019.12.4.03

Sabeeha S Shiny C, "ECG-Based Heartbeat Classification for Disease Diagnosis", Proceedings of the IEEE 2017 International Conference on Computing Methodologies and Communication, pp. 1113-1117, 2017.

Shalin Savalia and Vahid Emamian , "Cardiac Arrhythmia Classification by Multi-Layer Perceptron and Convolution Neural Networks,"MPDI bioengineering, vol. 5, 2018.

Wang, H., Shi, H., Chen, X. et al. An Improved Convolutional Neural Network Based Approach for Automated Heartbeat Classification. J Med Syst 44, 35 (2020). https://doi.org/10.1007/s10916-019-1511-2

Xin Gao , "Diagnosing Abnormal Electrocardiogram (ECG) via Deep Learning” April 2019. https://www. researchgate.net/publication/332348241_Diagnosing_ Abnormal_Electrocardiogram_ECG_via_Deep_ Learning 